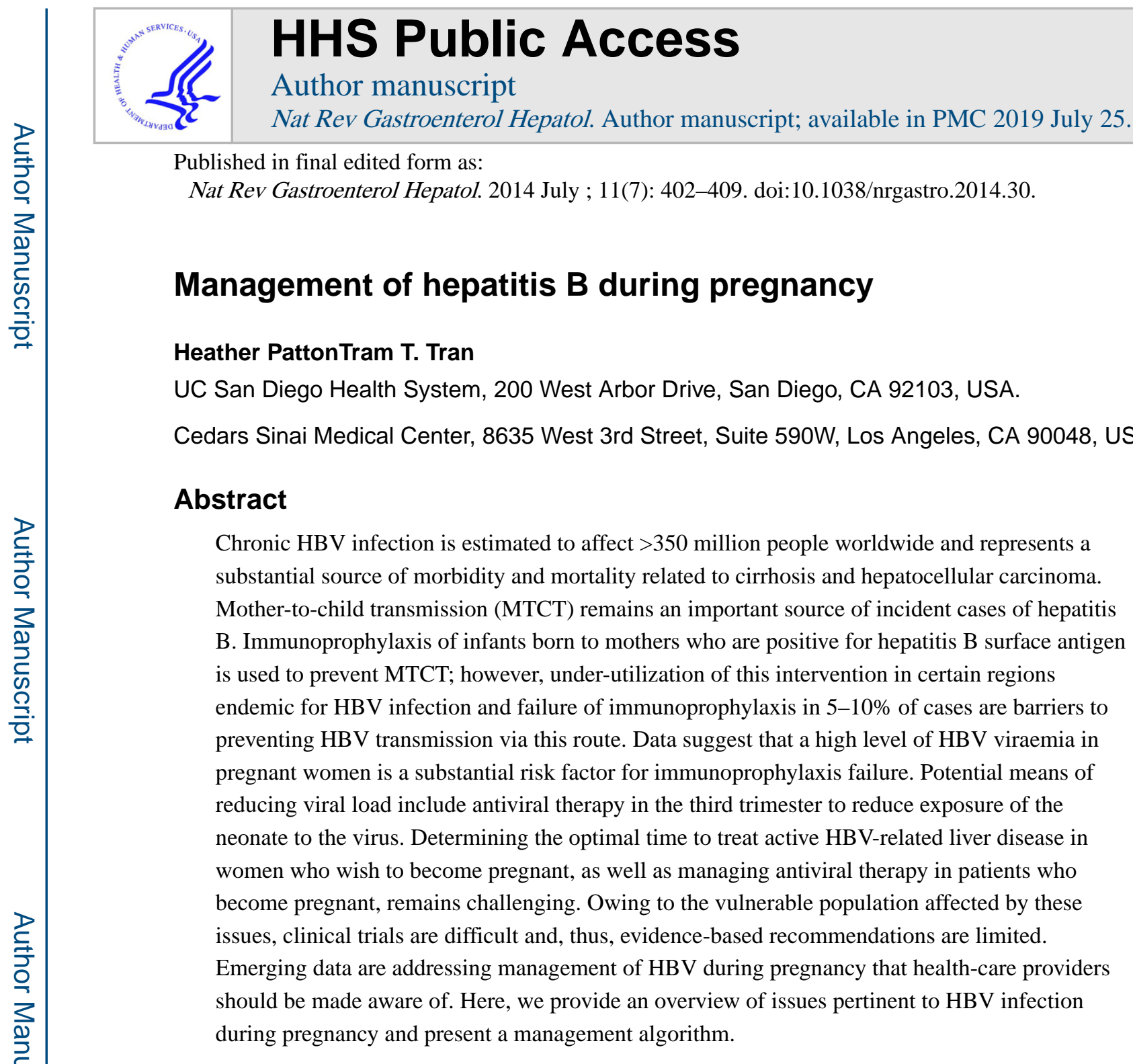

\title{
Introduction
}

Chronic HBV infection remains an important global cause of chronic liver disease and can result in cirrhosis, liver failure and/or hepatocellular carcinoma in as many as $15-40 \%$ of infected individuals. ${ }^{1}$ An estimated 2 billion people worldwide are thought to have been infected with HBV, 350-400 million of whom are chronically infected. An effective and safe vaccine against $\mathrm{HBV}$ is currently available. New data on $\mathrm{HBV}$ immunization rates in the USA from the National Health and Nutrition Examination Surveys (NHANES) demonstrate a marked increase in vaccination rates from $~ 30 \%$ in 1999 to $70-80 \%$ in $2010 .{ }^{2}$ However, 50 million new cases of hepatitis B continue to be diagnosed annually, with the largest burden of incident cases due to mother-to-child transmission (MTCT). ${ }^{3}$ In the USA, previously

Correspondence to: T.T.T. trant@cshs.org.

Author contributions

The authors contributed equally to all aspects in the production of this article.

Competing interests

T.T.T. acts as a consultant, advisor and/or speaker for Bristol-Myers Squibb, Gilead Sciences and Novartis. H.P. declares no competing interests. 
reported estimates of chronic HBV infection (1.25 million) have now been modified to 2.2 million when accounting for foreign-born individuals from regions with high endemic rates of seropositivity. ${ }^{3,4}$

Areas of the world endemic for HBV infection (defined as $8-15 \%$ of the population infected with HBV) include China, Southeast Asia, the Pacific Islands, sub-Saharan Africa, parts of the Middle East and the Amazon Basin. ${ }^{3}$ In these locations, MTCT is the most common route of infection and the lifetime risk of infection exceeds $60 \% .^{3}$ By contrast, regions not endemic for HBV ( $<2 \%$ of the population is infected with HBV; the USA, Australia and Western Europe) have primarily adult-to-adult transmission that is unlikely to result in chronic infection, and individuals have a low lifetime risk of infection $(<20 \%){ }^{3}$ In these regions, immigration patterns are important determinants of chronic HBV infection in the population and identification of $\mathrm{HBV}$ infection among women of childbearing age remains an important opportunity to reduce the future burden of disease.

Women of childbearing age pose a particular clinical concern in the transmission of hepatitis B as recent data suggest that high maternal HBV viraemia increases risk of HBV MTCT, even in the setting of appropriate and timely immunoprophylaxis. However, whether to initiate antiviral prophylaxis medications during pregnancy and the clinical dilemmas of when and how to initiate and/or discontinue medication is challenging.

\section{Routes of transmission}

HBV is transmitted via parenteral or mucosal exposure to infected body fluids; the highest viral concentrations are present in blood and serous fluids and the lowest concentrations present in saliva and semen. Routes of HBV transmission include sexual contact, parenteral drug use with shared paraphernalia, tattoos, piercings, acupuncture and needle-stick exposure in the health-care setting. In countries where HBV is endemic, most infections are acquired through perinatal exposure. Indeed, HBV MTCT rates up to 70-90\% have been reported in mothers who are $\mathrm{HBsAg}$ and $\mathrm{HBeAg}$ positive, and in the absence of postexposure prophylaxis in the infant. ${ }^{5}$ Perinatal exposure is also the route of transmission most likely to result in chronic HBV infection and is a vital target for reducing the burden of $\mathrm{HBV}$-related disease in populations endemic for HBV infection.

HBV can be transmitted via a vertical or horizontal route, but the risk of developing chronic HBV infection is strongly associated with the age of HBV exposure. The rate of chronic infection varies strikingly from $\sim 90 \%$ in exposed infants to $\sim 50 \%$ in toddlers and young children (aged $1-5$ years) and only $\sim 5 \%$ in exposed adults. ${ }^{6}$ Active-passive immunoprophylaxis with the hepatitis B vaccine and hepatitis B immune globulin (HBIG) also has a dramatic effect on MTCT rates, which vary markedly according to the mother's hepatitis B e antigen ( $\mathrm{HBeAg}$ ) status (70-90\% transmission rate for $\mathrm{HBeAg}$ - positive mothers versus $10-40 \%$ for $\mathrm{HBe} A g$-negative mothers) if no immunoprophylaxis is provided. 5

The standard active-passive immunoprophylaxis regimen involves administration of HBIG and hepatitis B vaccination immediately after birth (within $12 \mathrm{~h}$ ) to infants of hepatitis B 
surface antigen (HBsAg)-positive mothers, followed by two additional doses of vaccine within 6-12 months. This regimen prevents transmission of HBV infection in $~ 95 \%$ of cases. ${ }^{7}$ However, a systematic review of literature published from 1975-2011 determined that this approach fails to prevent HBV transmission in 8-30\% of children who are born to highly viraemic mothers. ${ }^{8}$ Postulated causes of immunoprophylaxis failure include high levels of maternal viraemia, intrauterine infection or mutation of HBsAg. ${ }^{9-13}$ Thus, a clinical need exists to identify all causes of immunoprophylaxis failure and to determine safe and effective means of reducing MTCT rates to zero.

\section{Risk factors for MTCT}

Women of childbearing age who are infected with HBV tend to be in the immune-tolerant or immune-active phases of chronic HBV infection and have high levels of viraemia. As such, pregnancy and the perinatal period present the most critical time period in which the future burden of chronic HBV infection might be affected. ${ }^{14}$ In recognition of the importance of identifying HBV infection during pregnancy to reduce incident HBV infections, current recommendations by the US Preventive Services Task Force are for universal HBsAg screening of all pregnant women. ${ }^{15}$ Outside the screening recommendation for all pregnant women, the AASLD (American Association for the Study of Liver Diseases) guideline recommendations for the general population state that screening should be limited to certain high-risk populations, such as individuals who are born in areas of high or intermediate HBV prevalence, persons who have injected drugs, household or sexual contacts of HBsAgpositive persons, and persons who need immunosuppressive therapy. ${ }^{16}$

MTCT of HBV can take place intrauterine or during the peripartum or postpartum periods. Intrauterine infection has been defined as detectable HBsAg in infants in the first 30 days following delivery or detectable HBV DNA in peripheral blood samples taken from the neonate. ${ }^{17}$ Provocative data from China have reported HBsAg staining in ovarian follicular and placental capillary endothelial cells as a risk factor for intrauterine transmission. ${ }^{18}$ Other potential means of MTCT in the antepartum period include passage via maternal peripheral blood mononuclear cells or trans-placental leakage causing maternal-fetal microcirculation (related to prolonged uterine contractions). Among studies reporting risk factors for antepartum transmission, some have indicated increased risk of threatened abortion, whereas amniocentesis has not been found to increase the risk of HBsAg or HBV DNA transfer to the neonate. ${ }^{18-23}$ In the past year, however, an association between high maternal viraemia and HBV transmission to the neonate with amniocentesis has been reported. ${ }^{24}$ Further studies are needed before changes to recommendations should be considered. During vaginal delivery, direct contact with fluid in the maternal genital tract might result in MTCT. Indeed, high rates of HBs Ag have been detected in vaginal fluids, and detection of HBsAg in the gastric lavage of newborn babies is associated with an increased risk of the infant being HBs Ag-positive at 3 months of age. ${ }^{25}$ In the postpartum period, breastfeeding has been evaluated as a potential source of MTCT (either via HBsAg in breast milk or through exposure to infected blood via abrasions on the nipple), but has not been demonstrated to pose a substantial risk of $\mathrm{HBV}$ transmission to infants who have received appropriate immunoprophylaxis. ${ }^{26}$ Current recommendations from the Centers for Disease Control and Prevention support breastfeeding in mothers with HBV infection. ${ }^{7}$ With the exception of 
lamivudine, limited information is available regarding the excretion of antiviral agents into human breast milk. ${ }^{27}$ Prescribing information for all anti-HBV antiviral agents (including tenofovir disoproxil fumarate [TDF], telbivudine and entecavir) recommends against breastfeeding while taking these agents.

Maternal HBeAg positivity is a well-known risk factor for MTCT. HBeAg is believed to have an immuno-regulatory function and, clinically, is indicative of viral replication and increased risk of transmission. ${ }^{28}$ It is not clear from the available data if or how $\mathrm{HBe} \mathrm{Ag}$ positivity affects perinatal transmission independently of viral load, although data from China on >1,000 HBs Ag- positive mothers have reported both HBeAg and HBV DNA positivity to be independent predictors of immune-prophylaxis failure. ${ }^{29-31}$ Maternal HBe Ag is believed to be transferred in utero through the placenta where it interferes with T-cell function, thereby inducing T-cell tolerance of HBV in the infant. ${ }^{32}$

Maternal viraemia has been extensively documented as one of the strongest risk factors for the transmission of HBV in pregnancy. In a large, nested case-control study of $773 \mathrm{HBsAg}$ positive women in Taiwan, high levels of HBV DNA ( $\geq 1.4 \mathrm{ng} / \mathrm{ml}$ ) in HBeAg-positive women were associated with persistent infection in infants (odds ratio [OR] 147). ${ }^{33}$ Even in the era of immunoprophylaxis, high viraemia remains a strong predictor of MTCT. In a study of 138 babies born to HBsAg-positive women, Wiseman et al. reported an immunoprophylaxis failure rate of $9 \%$ among infants born to woman with high HBV viraemia. Of note, immunoprohylaxis failure only occurred in infants born to $\mathrm{HBeAg}$ positive women who had HBV DNA levels $\geq 8 \log _{10}$ copies per ml. ${ }^{34}$ Literature published since 2012 also addresses the magnitude of MTCT risk according to varying thresholds of maternal HBV DNA. ${ }^{35}$ These data are particularly useful for counselling mothers about the likelihood of immunoprophylaxis failure (Figure 1). ${ }^{35}$

\section{Reducing MTCT}

Controversy exists as to whether infants born via caesarean section have a lower risk of HBV MTCT than those born vaginally. Although some data have now been reported to support elective caesarean section to reduce MTCT, including a meta-analysis that suggested a $17.5 \%$ absolute risk reduction when compared with immunoprophylaxis alone, ${ }^{36}$ other studies have found that elective caesarean section offers no benefit. ${ }^{37,38}$ Data from Beijing reporting on 1,409 infants born to HBsAg- positive mothers from 2007-2011, all of whom received appropriate immunoprophylaxis at birth, reported MTCT rates of $1.4 \%$ after elective caesarean section compared with $3.4 \%$ after vaginal delivery and $4.2 \%$ after emergency caesarean section $(P<0.05) .{ }^{38}$ When mothers in this study were stratified according to their level of HBV DNA, delivery modality did not affect MTCT rates among those with lower levels of viraemia (defined as $<6 \log _{10}$ copies per $\mathrm{ml}$ ). Thus, these data suggest a potential role for elective caesarean section among women with high HBV DNA levels. Before definitive guidelines can be created, validation studies are needed and further data are required to determine the relative safety and efficacy of elective caesarean section versus antiviral therapy during pregnancy in reducing MTCT rates without compromising fetal outcomes. 
Regardless of delivery modality, postpartum management must include administration of HBIG and hepatitis B vaccine to the neonate within $12 \mathrm{~h}$ of birth, as the efficacy of the vaccine declines as the time between birth and initial dose administration increases. As in other populations, it is recommended that infants receive a total of three doses of the hepatitis B vaccine (one at birth, the second at 4-8 weeks of age and the third at 6 months). ${ }^{7}$ It is also advised that the titres of HBsAg and antibodies against HBsAg be assessed at 9-15 months of age. Although much of this Review is focused on the means to address immunoprophylaxis failure, this immunoprophylaxis regimen, when properly administered, is highly effective for the prevention of MTCT (protecting 95-100\% of at-risk newborn babies). However, challenges still exist in the implementation of the appropriate administration of this regimen and doses vary according to geographic location and healthcare system. In regions where chronic HBV infection prevalence has historically been high ( $\geq 8 \%$ ), such as sub-Saharan Africa and Southeast Asia, only $42 \%$ of countries reported the dose of hepatitis B vaccine given at birth in immunization schedules. ${ }^{39}$ Data from the Hepatitis B Research Network on 482 live births have revealed marked education gaps among North American mothers infected with HBV, 13\% of whom did not know the vaccination status of their child and $27 \%$ of whom did not know whether their child had received HBIG. $^{40}$

\section{Antiviral therapy}

\section{Women of childbearing age}

Women of childbearing age who are infected with HBV present unique clinical dilemmas given the potential of HBV to cause chronic liver disease, to flare during or after pregnancy, and to be transmitted during the perinatal period. If a woman of childbearing age presents with chronic $\mathrm{HBV}$ infection and is currently not pregnant, goals of management are to determine the state of her liver disease and the necessity for antiviral therapy within the context of her family planning status. Indications for medical treatment of chronic HBV infection are based upon severity of disease as judged by HBV DNA level, HBeAg status and evidence of liver injury (by alanine aminotransferase [ALT] level and/or liver histology). ${ }^{16}$ Often women of childbearing age will be in the immune-tolerant stage of disease, characterized by a serological profile of $\mathrm{HBsAg}$-positivity, $\mathrm{HBeAg}$-positivity and very high levels of viraemia ( $>10^{8}$ copies per ml) with persistently normal serum ALT levels ( $<19$ IU/ $\mathrm{ml})$. According to the AASLD practice guidelines, ${ }^{16}$ these women would not be candidates for antiviral therapy. However, given the high maternal levels of viraemia during the immune-tolerant phase, antiviral therapy might be indicated to reduce perinatal transmission during later stages of pregnancy (discussed below).

If a woman is planning pregnancy in the long-term future ( $>18$ months) and is a candidate for antiviral treatment, interferon therapy can be considered, as this modality constitutes a defined treatment course of 48 weeks (unlike oral antiviral HBV therapy, which typically requires long-term treatment). Unfortunately, response rates with interferon are suboptimal and this therapy is associated with considerably greater adverse effects than oral antiviral agents. Additionally, candidates for interferon therapy must be willing and able to take 
contraceptive measures throughout therapy and for a 6-month washout period after therapy has concluded.

By contrast, women planning pregnancy in the immediate future (before the duration of any interferon treatment and washout period ends) are unlikely to be initiated on antiviral therapy due to concerns regarding fetal exposure to these drugs in early pregnancy. Nucleoside or nucleotide antiviral therapy has the potential to promote mitochochondrial toxicity and is of unclear benefit in young patients who have no clear evidence of advanced liver disease. ${ }^{16}$ In patients who are suspected of having more advanced fibrosis and/or cirrhosis or evidence of highly active disease (based on elevated levels of ALT and/or histology), the clinical risk-benefit assessment might favour initiating therapy and continuing therapy throughout the course of pregnancy. Pregnancy itself can be associated with mild hepatitis flares, but rarely more severe flares (defined as ALT levels $>10$ times the upper normal limit). In the rare circumstance in which a flare occurs and if evidence of hepatic decompensation exists, initiation of antiviral therapy might be necessary to avoid untoward outcomes for both the mother and fetus. ${ }^{41}$ The highest risks of HBV-associated decompensation in pregnancy are seen in women who have underlying cirrhosis, being reported at a rate of $15 \%$ in a retrospective review of 399 mothers with cirrhosis, and associated with $1.8 \%$ risk of mortality for mothers and $5.2 \%$ risk of fetal mortality. ${ }^{42}$

Women who have previously received antiviral therapy for chronic HBV infection and who then present for care in early pregnancy require thoughtful evaluation to weigh the risks of antiviral discontinuation against those of exposure to nucleoside or nucleotide agents during the first trimester. The most important variable to consider in these cases is the severity of the underlying liver disease at the time antiviral therapy was initiated. Some clinicians might choose to continue therapy, and, in that case, TDF is often considered the long-term therapy of choice throughout pregnancy owing to the safety data available in patients with HIV, potency and low risk of resistance developing. Women presenting in the second or third trimester of pregnancy who have been taking antiviral therapy risk the possibility of rebound viral hepatitis with drug discontinuation as well as an increased risk of MTCT with high HBV DNA levels. Thus, expert opinion would favour continuation of antiviral therapy in this scenario. One small series of women $(n=12)$ who discontinued antiviral therapy during pregnancy reported viral rebound in $67 \%$ of patients, with a fivefold increase in ALT levels observed in $50 \%$ of patients. ${ }^{43}$ Fortunately, spontaneous recovery was seen in all cases, but this report does highlight the potential risk of discontinuing antiviral therapy in pregnant women who have advanced fibrosis. ${ }^{43}$

Finally, women who are initially diagnosed with HBV through screening performed in the first trimester of pregnancy warrant assessment for evidence of underlying liver disease. If the patient has previously unrecognized advanced liver disease, then initiating therapy might be warranted as the unknown risk of antiviral therapy to the fetus would be estimated to be lower than the risk of hepatic decompensation during pregnancy (reported at $14 \%$ in one retrospective study and associated with an increased risk of maternal and fetal mortality, as noted earlier). ${ }^{44}$ However, most women of childbearing age will be in the immune-tolerant phase or have early disease, in which case antiviral therapy will be deferred, except in 
women with high HBV viraemia, for whom initiation of antiviral therapy might be advised in the third trimester for purposes of reducing risk of MTCT.

\section{Prevention of MTCT}

If a woman does not have active disease, advanced fibrosis or cirrhosis (each of which qualify her as a candidate for HBV therapy), then, as the third trimester approaches, consideration of the risk of perinatal hepatitis B transmission to the newborn baby becomes the predominant clinical concern. Ideally, conversations about the risk of MTCT and means of reducing this risk should occur in the antepartum period or early during the first trimester so that women have adequate time to consider management options with their hepatologist and obstetrician. Seven therapies are currently approved for the treatment of HBV infection (summarized in Table 1). Interferon therapy is best used in select women prior to the time that they are planning pregnancy as a means to reduce risk of MTCT (via HBeAg seroconversion with markedly reduced viral load) and should be avoided in women who are currently pregnant or who wish to become pregnant in the near term (within 18 months). Although only two of the currently approved therapies (TDF and telbivudine) are categorized as FDA category B drugs (Table 1), a growing number of reports have detailed clinical experience using these drugs to reduce MTCT. In addition, drugs such as lamivudine and TDF, which are used for HIV therapy, have also been administered to pregnant women with HIV, enabling further clinical observations of safety.

The largest experience of patients with HBV infection treated with lamivudine to reduce MTCT is included in a meta-analysis published in $2011 .{ }^{45}$ This analysis included 15 randomized controlled trials (RCTs) with a total of 1,693 HBV-infected mothers, most of whom were identified through the China Biological Medicine Database (published in Chinese). Lamivudine therapy (initiated at week 28 of gestation and beyond) was found to substantially reduce the rate of MTCT compared with immunoprophylaxis alone (as determined by HBsAg or HBV DNA measurement in newborn babies or infants). However, as only two of the 15 studies were methodologically of high quality, the remainder judged to be of low quality by the authors of the meta-analysis, these results should be regarded with caution.

In the largest RCT of lamivudine to reduce MTCT, Xu et al. ${ }^{46}$ treated 150 pregnant women with lamivudine (100 mg per day) or placebo if they had HBV DNA levels $>1,000 \mathrm{MEq} / \mathrm{ml}$ (where $1 \mathrm{MEq} \sim=10^{6}$ copies per $\mathrm{ml}$ ) starting at week 32 of gestation to 4 weeks postpartum (Table 2). Data from this RCT are limited in that infants born to 26 women in the lamivudine arm received vaccination but not HBIG at birth and the dropout rate was high. An intentionto-treat analysis found an MTCT rate of $18 \%$ with lamivudine versus $39 \%$ with placebo ( $P=$ 0.014), despite the noted methodological problems. A later report in which lamivudine therapy was given in the second or third trimester included treatment-naive mothers who were HBeAg-positive with HBV DNA levels $>6 \log _{10}$ copies per $\mathrm{ml}(n=155) .{ }^{47}$ All infants in this study received standard active-passive immunoprophylaxis and cases were compared with 89 matched controls with similar virological profiles who were not treated with antiviral therapy during pregnancy. The MTCT rate was $0 \%$ in the lamivudine-treated group 
versus $5 \%$ in the control group $(P=0.012)$. No differences in infant complications or adverse events were seen with or without antiviral therapy. ${ }^{47}$

Han et al. ${ }^{48}$ conducted a prospective, open-label trial of 229 women aged 20-40 years who were HBeAg-positive with HBV DNA levels $>7 \log _{10}$ copies per ml. All women were offered antiviral therapy, the 135 who accepted received telbivudine ( $600 \mathrm{mg}$ daily) from gestation week 20-32. The comparison arm consisted of 94 women who consented to participate in the trial but declined antiviral therapy. All infants were administered appropriate immunoprophylaxis. Mean viral load at enrolment was $\sim 8 \log _{10}$ copies per $\mathrm{ml}$ in both arms, which reduced to $2.44 \log _{10}$ copies per $\mathrm{ml}$ in the telbivudine-treated arm prior to delivery. The reported MTCT rate was $0 \%$ in infants born to the women who received telbivudine therapy compared with $8 \%$ in infants born to the women who received no antiviral therapy. One infant in each group had low birth weight and six infants in the telbivudine group (compared with five infants in the control group) developed pneumonia by age 7 months; no congenital abnormalities were identified in either group. ${ }^{48}$ This study adds to the body of evidence that reduction of maternal viraemia reduces MTCT, although the choice of telbivudine is still limited by lack of human safety data when com-pared with TDF. $^{49}$ In a similar study, Pan and colleagues compared 53 women with HBeAg-positive HBV infection (viral loads $>6 \log _{10}$ copies per $\mathrm{ml}$ ) and elevated ALT levels who had telbivudine therapy initiated in the second or third trimester with 35 similar women who declined therapy following a discussion of risks and benefits. The immunoprophylaxis failure rate in this study was $0.0 \%$ for the telbivudine therapy group compared with $8.6 \%$ in the group who declined telbivudine, with no marked difference in the rates of adverse events at a follow-up of 28 weeks postpartum. ${ }^{50}$

Few data exist to support treatment early in the course of pregnancy for prevention of MTCT (Table 2). One study from Beijing evaluated 86 women who began telbivudine therapy during the antenatal period or in the first trimester $(86 \% \mathrm{HBeAg}$-positive and $2.3 \%$ cirrhotic). ${ }^{51}$ Spontaneous abortions were observed in $7.9 \%$ of the women treated with telbivudine in early pregnancy, which was not felt by the authors of the study to be greater than expected. Adverse events, reported in $>5 \%$ of mothers in the perinatal period, included meconium staining of the amniotic fluid (10\%), premature birth (8\%) and premature rupture of membranes $(6 \%)$, which were also not felt to be greater than expected. The reported congenital abnormality rate (with postpartum follow-up duration 6-24 months) was 3.8\% and there were no cases of MTCT. ${ }^{51}$ Further pregnancy outcome data are required before the safety of antiviral therapy exposure in the first trimester of pregnancy can be determined. Longer-term follow-up data for infants exposed to telbivudine in utero have been reported in 200 pregnant women who were treated with telbivudine from 20-32 weeks gestation-202 infants born to these women were followed-up for a minimum of 1 year (maximum followup 4 years). ${ }^{52}$ The MTCT rate was $0.0 \%$ in this study; two congenital abnormalities were identified $(0.1 \%)$, one at 1.5 years of age (megacolon) and another at 2 years of age (patent ductus arteriosus). ${ }^{52}$ At this time, not enough data exist to support early treatment of HBV to prevent MTCT.

Although robust data are available from studies in the HIV-infected TDF-exposed pregnant population, considerably fewer published data have assessed the efficacy of TDF therapy in 
reducing MTCT risk in HBV-infected pregnant women. In one small series of women ( $n=$ 11) with HBeAg-positive disease and high viraemia ( $\geq 10^{6}$ copies per ml) treated with TDF at a median of 29 weeks gestation for a median of 10 weeks prior to delivery, a $0 \%$ immunoprophylaxis failure rate was reported and no adverse events were identified in either mothers or their infants. ${ }^{53} \mathrm{~A}$ larger $(n=48)$ retrospective review of women who were treated with TDF throughout pregnancy reported a MTCT rate of $0 \%$, with a spontaneous abortion rate of $6 \%$ in the first trimester. ${ }^{54}$ The observed fetal adverse event rates were also low (3.6\% for each of the following: preterm labour, prolonged labour, anterior placenta and meconium staining) ${ }^{54}$ Finally, in a multi-centre, prospective study from Australia, 60 woman with HBV DNA levels $>10^{7} \mathrm{IU} / \mathrm{ml}$ commencing therapy with TDF at 32 weeks gestation were compared with women treated with lamivudine $(n=21)$ from the same time point in pregnancy and untreated historical controls $(n=9) .5534$ babies born to woman taking TDF had a follow-up at 9 months and, at this time, no infants were HBsAg-positive; no evidence of congenital abnormalities or abnormal growth parameters was observed. ${ }^{55}$ Thus, although the published experience is small for TDF, it seems to be highly effective at reducing MTCT risk and does not seem to be associated with high rates of congenital abnormalities.

The Antiretroviral Pregnancy Registry (APR) is another source of data regarding the safety of HBV therapy during pregnancy. This voluntary, prospective, observational database of women taking antiretroviral therapies during pregnancy was designed to detect early signals of teratogenicity associated with these medications. ${ }^{49}$ As of January 2013, 15,877 women were enrolled in the APR, with a minority reporting HBV monoin-fection $(n=233)$ or HIVHBV co-infection $(n=163)$. Lamivudine was associated with a $3.2 \%$ birth defect rate (135 out of 4,273) after exposure in the first trimester and a 2.8\% birth defect rate (198 out of $6,989)$ after exposure in the second or third trimester. Exposure to TDF in the first trimester was associated with a $2.3 \%$ birth defect rate $(42$ out of 1,800$)$ and a $2.2 \%$ birth defect rate (20 out of 894) when TDF therapy was initiated in the second or third trimester. In comparison, the Centers for Disease Control and Prevention report rates of birth defects to be $\sim 3 \%$ in the general USA population. ${ }^{49}$

In summary, a growing body of literature supports both the safety and efficacy of antiviral therapy initiated in late pregnancy for reduction of MTCT among women whose children are at highest risk of immunoprophylaxis failure (HBV DNA levels $\geq 10^{7}$ copies per ml). Although some studies have suggested a favourable safety profile for antiviral therapy even in the first and second trimesters of pregnancy when used purely for the purposes of reducing MTCT, antiviral therapy can be initiated in the third trimester, thereby minimizing the risk associated with fetal exposure to these medications. ${ }^{56}$ Treatment at HBV DNA levels $<10^{6}$ copies per $\mathrm{ml}$ does not seem to be indicated unless the mother has existing liver disease, in which case viral suppression is recommended. The end point of antiviral therapy administered to reduce the risk of MTCT is typically in the immediate postpartum period for mothers who plan to breastfeed their infants. Discontinuation of therapy at any point during or after pregnancy requires careful monitoring due to the potential for flares upon withdrawal (discussed below). 


\section{HBV infection and pregnancy outcomes}

Limited data are available to address whether HBV infection affects obstetric outcomes independently of other variables. A retrospective analysis of all births in Florida ( $n=$ 1,670,369) from 1998 to 2007 for pregnancy outcomes (preterm birth, low birth weight, small for gestational age, fetal distress, neonatal jaundice and congenital abnormalities) according to $\mathrm{HBV}$ or $\mathrm{HCV}$ monoinfection or co-infection found that women with $\mathrm{HBV}$ infection were less likely to have infants born small for gestational age (OR 0.79; 95\% CI 0.66-0.95) compared with the uninfected cohort. ${ }^{57}$ This finding indicates that HBV infection does not substantially increase the risk of adverse outcomes in pregnancy compared with individuals without $\mathrm{HBV}$ or $\mathrm{HCV}$ infection. A retrospective case-control study addressing this question compared $253 \mathrm{HBsAg}$ carriers who had a singleton pregnancy with 253 controls matched for age, parity and year of delivery. HBsAg carriers had increased rates of antepartum haemorrhage, gestational diabetes and threatened preterm labour. ${ }^{58}$ The most recent publication to address this question was published in 2013 and found that $\mathrm{HBsAg}$-positivity was associated with a reduced risk of pregnancy-induced hypertension (adjusted OR 0.79; 95\% CI 0.66-0.95) and pre-eclampsia (adjusted OR 0.71; 95\% CI 0.56$0.91) .59$ Thus, from currently available data it is difficult to state with any confidence that HBsAg-positive mothers face an increased risk of obstetric complications attributable to their infection status.

\section{Postpartum flares of hepatitis}

Owing to immunological changes in pregnancy, flares of hepatitis are observed both during and after pregnancy, although the true incidence of pregnancy-related disease flares and risk factors are poorly understood. The Hepatitis B Research Network ${ }^{40}$ reported the rate of hepatitis flare (defined as ALT >200 IU/ml) in 82 women after giving birth to be fairly low (2.4\%). ${ }^{60}$ In a cohort of 126 Australian women recruited during early pregnancy, hepatitis flare (defined as ALT twice the upper limit of normal or twice the baseline value if this level was elevated) was reported in $21 \%$ of women. ${ }^{61}$ Six women in this cohort took antiviral therapy in pregnancy to reduce MTCT rate. Risk factors identified were $\mathrm{HBeAg}$ - positivity (OR 2.19; 95\% CI 1.13-4.22) and first pregnancy (OR for parity 0.56 ; 95\% CI $0.37-0.85$ ). ${ }^{61}$ Data evaluating how the postpartum duration of antiviral therapy affects the risk of postpartum flare of hepatitis amongst mothers with HBV DNA levels $\geq 7 \log _{10} \mathrm{IU} / \mathrm{ml}$ found no difference in women treated for $\leq 4$ weeks, $>4$ weeks, or women not treated with antiviral therapy at all. Thus, whether antiviral therapy in pregnancy has a significant effect on postpregnancy flare risk is currently unclear. ${ }^{62}$

\section{Conclusions}

HBV transmission remains a global health issue despite the availability of an effective and safe vaccine. Further implementation of HBV vaccination and administration of HBIG at birth in countries highly endemic for HBV is important. However, although HBIG and the HBV vaccine are highly effective, a high level of maternal HBV DNA (especially $>10^{8} \mathrm{log}$ copies per $\mathrm{ml}$ or $2 \times 10^{7} \mathrm{IU} / \mathrm{ml}$ ) is a strong predictor of immunoprophylaxis failure and results in an $\sim 8 \%$ risk of $\mathrm{HBV}$ transmission to the neonate. Consideration of antiviral 
therapy to reduce viral load in the third trimester of pregnancy can be considered in this high-risk group, but a careful discussion with the mother about the risks, benefits and limitations of the current data is essential. For highly viraemic mothers, an alternative consideration to reduce risk of immunoprophylaxis failure includes elective caesarean section, although conflicting data suggest more studies are needed before changes in the mode of birth can be recommended. Thus, the decision regarding interventions to reduce MTCT of HBV in this subset of women requires an informed discussion with both her hepatology and obstetrics providers. Finally, any mothers who have advanced disease (especially cirrhosis) are at risk of severe flares of disease during and after pregnancy if antiviral therapy is discontinued, which can be fatal in rare cases. Thus, discontinuation of antiviral therapy is not recommended in these women.

\section{References}

1. Bosch FX et al. Epidemiology of hepatocellular carcinoma. Clinic Liver Dis 9, 191-211 (2005).

2. WHO Fact Sheets. Hepatitis B [online], http://who.int/inf-fs/en/fact204.html (2000).

3. Kowdley KV et al. Prevalence of chronic hepatitis B among foreign born persons living in the United States by country of origin. Hepatology 56, 422-433 (2012). [PubMed: 22105832]

4. Devaki $P$ et al. Changes in hepatitis $B(\mathrm{HBV})$ vaccination rates and differences in various general demographics across National Health and Nutrition Examination Surveys (NHANES) from 1999_ 2010. Hepatology 58 (Suppl.), 607A-608A (2013).

5. Stevens $\mathrm{CE}$ et al. $\mathrm{HBeAg}$ and anti-HBe detection by radioimmunoassay: correlation with vertical transmission of hepatitis B virus in Taiwan. J. Med. Virol 3, 237-241 (1979). [PubMed: 479860]

6. Edmunds WJ et al. The influence of age on the development of the hepatitis B carrier state. Proc. R. Soc. B 253, 197-201 (1993).

7. US Department of Health and Human Services. MMWR: a comprehensive immunization strategy to eliminate transmission of hepatitis B virus infection in the United States [online], http:// www.cdc.gov/mmwr/preview/mmwrhtml/rr5416a1.htm (2005).

8. Pan CQ et al. An algorithm for risk assessment and intervention of mother to child transmission of hepatitis B. Clin. Gastroenterol. Hepatol 10, 452-459 (2012). [PubMed: 22079509]

9. Stevens CE et al. Vertical transmission of hepatitis B antigen in Taiwan. N. Engl. J. Med 292, 771774 (1975). [PubMed: 1113797]

10. Ghaziasadi A et al. Mutational analysis of HBsAg-positive mothers and their infected children despite immunoprophylaxis. Iran J. Allergy Asthma Immunol 12, 352-360 (2013). [PubMed: 23996711]

11. Lee SD et al. Maternal hepatitis B virus DNA in mother-infant transmission. Lancet 1, 719 (1989).

12. Ngui SL et al. Low detection rate and maternal provenance of hepatitis B virus $S$ gene mutants in cases of failed postnatal immunoprophylaxis in England and Wales. J. Infect. Dis 176, 1360-1365 (1997). [PubMed: 9359739]

13. Karthigesu VD et al. A hepatitis B virus variant found in the sera of immunised children induces a conformational change in the HBsAg "a" determinant. J. Med. Virol 58, 346-352 (1999). [PubMed: 10421400]

14. Chiang CJ et al. Thirty-year outcomes of the National Hepatitis B Immunization Program in Taiwan. JAMA 310, 974-976 (2013). [PubMed: 24002285]

15. US Preventive Services Task Force. Screening for hepatitis B virus infection in pregnancy: US Preventive Services Task Force reaffirmation recommendation statement. Ann. Intern. Med 150, 869-873 (2009). [PubMed: 19528565]

16. Lok AS \& McMahon BJ AASLD practice guideline update chronic hepatitis B: update 2009. Hepatology 50, 1-36 (2009). [PubMed: 19554618]

17. Bai GQ, Li SH, Yue YF \& Shi L The study on role of peripheral blood mononuclear cell in HBV intrauterine infection. Arch. Gynecol. Obstet 283, 317-321 (2011). [PubMed: 20107823] 
18. Yu M et al. Correlation between vertical transmission of hepatitis B virus and the expression of HBsAg in ovarian follicles and placenta. PLoS ONE 8, e5426 (2013).

19. Lin HH et al. Transplacental leakage of HBeAg- positive maternal blood as the most likely route in causing intrauterine infection with hepatitis B virus. J. Pediatr 111, 877-881 (1987). [PubMed: 3681555]

20. Lazizi Y, Badur S \& Perk Y Selective unresponsiveness to HBsAg vaccine in newborns related with an in utero passage of hepatitis B virus DNA. Vaccine 15, 1095-1100 (1997). [PubMed: 9269052]

21. Zhang SL et al. Mechanism of Intrauterine infection of hepatitis B virus. World J. Gastroenterology $10,437-438$ (2004).

22. Xu DZ et al. Risk factors and mechanism of transplacental transmission of hepatitis B virus: a case-control study. J. Med. Virol 67, 20-26 (2002). [PubMed: 11920813]

23. Towers CV, Asrat T \& Rumney $\mathrm{P}$ The presence of hepatitis B surface antigen and deoxyribonucleic acid in amniotic fluid and cord blood. Am. J. Obstet. Gynecol 184, 1514-1518 (2001). [PubMed: 11408875]

24. Yi W et al. Risk of hepatitis B virus vertical transmission after amniocentesis in mothers with chronic hepatitis B. J. Hepatol 10.1016/j.jhep.2013.11.008.

25. Wong VC, Lee AK \& Ip HM Transmission of hepatitis B antigens from symptoms free carrier mothers to the fetus and the infant. Br. J. Obstet. Gynaecol 87, 958-965 (1980). [PubMed: 7437368]

26. Hill JB et al. Risk of hepatitis B transmission in breast-fed infants of chronic hepatitis B carriers. Obstet. Gynecol 99, 1049-1052 (2002). [PubMed: 12052598]

27. Mirochnik M et al. Antiretrovial concentrations in breast-feeding infants of mothers receiving highly active antiretroviral therapy. Antimicrob. Agents Chemother 53, 1170-1176 (2009). [PubMed: 19114673]

28. Milich D \& Liang TJ Exploring the biological basis of hepatitis B e antigen in hepatitis B virus infection. Hepatology 38, 1075-1086 (2003). [PubMed: 14578844]

29. Wang ZH et al. Quantitative analysis of HBV DNA level and HBeAg titer in hepatitis B surface antigen positive mothers and their babies: $\mathrm{HBeAg}$ passage through the placenta and the rate of decay in babies. J. Med. Virol 71, 360-366 (2003). [PubMed: 12966540]

30. Guo Z et al. Risk factors of HBV intrauterine transmission among HBsAg-positive pregnant women. J. Viral Hepatol 20, 317-321 (2013).

31. Yin $Y$ et al. Identification of risk factors associated with immunoprophylaxis failure to prevent the vertical transmission of hepatitis B virus. J. Infect 66, 447-452 (2013). [PubMed: 23286968]

32. Tse $\mathrm{K}$ et al. Immunoprophylaxis of babies borne to hepatitis B carrier mothers. Hong Kong Med. J 12, 368-374 (2006). [PubMed: 17028357]

33. Burk RD et al. Outcome of perinatal hepatitis B virus exposure is dependent on maternal virus load. J. Infect. Dis 170, 1418-1423 (1994). [PubMed: 7995980]

34. Wiseman E et al. Perinatal transmission of hepatitis B virus: an Australian experience. MJA 109, 489-492 (2009).

35. Zou $\mathrm{H}$ et al. Virologic factors associated with failure to passive-active immunoprophylaxis in infants born to HBsAg-positive mothers. J. Viral Hepatol 19, e18-e25 (2012).

36. Yang $\mathrm{J}$ et al. Elective caesarean section versus vaginal delivery for preventing mother to child transmission of hepatitis B virus-a systematic review. Virol. J 5, 100 (2008). [PubMed: 18755018]

37. $\mathrm{Hu} \mathrm{Y}$ et al. Effect of elective cesarean section on the risk of mother-to-child transmission of hepatitis B virus. BMC Pregnancy Childbirth 13, 119 (2013). [PubMed: 23706093]

38. Pan CQ et al. Cesarean section reduces perinatal transmission of HBV Infection from hepatitis $B$ surface antigen-positive women to their infants. Clin. Gastroenterol. Hepatol 10.1016/j.cgh. 2013.04.026.

39. Centers for Disease Control and Prevention (CDC) Implementation of newborn hepatitis B vaccination-worldwide, 2006. MMWR Morb. Mortal. Wkly Rep 46, 1249-1252 (2006). 
40. Tran TT et al. Hepatitis B Research Network (HBRN): maternal knowledge of children's hepatitis B infection and vaccination status [abstract]. Hepatology 58 (Suppl.), 622A-623A (2013).

41. Singhal A et al. Chronic HBV with pregnancy: reactivation flare causing fulminant hepatic failure. Annals Hepatol 10, 233-236 (2011).

42. Shaheen AA \& Myers RP The outcomes of pregnancy in patients with cirrhosis: a populationbased study. Liver Int 30, 275-283 (2010). [PubMed: 19874491]

43. Kim HY et al. Outcome after discontinuing antiviral agents during pregnancy in women infected with hepatitis B. J. Clin. Virol 56, 299-305 (2013). [PubMed: 23273664]

44. Nguyen $G$ et al. Clinical course of hepatitis B virus infection during pregnancy. Aliment. Pharmacol. Ther 29, 755-764 (2009). [PubMed: 19183158]

45. Han L et al. A meta-analysis of lamivudine for interruption of mother-to-child transmission of hepatitis B virus. World J. Gastroenterol 17, 4321-4333 (2011). [PubMed: 22090789]

46. Xu WM et al. Lamivudine in late pregnancy to prevent perinatal transmission of hepatitis B virus infection: a multicentre, randomized, double-blind, placebo-controlled study. J. Viral Hepat 16, 94-103 (2009). [PubMed: 19175878]

47. Yi W, Liu M, Chen A \& Pan C The efficacy of lamivudine use in the second vs. third trimester of pregnancy in preventing vertical transmission of HBV in highly viremic mothers [abstract]. Hepatology 58 (Suppl) 614A (2013).

48. Han G-R et al. A prospective and open label study for the efficacy and safety of telbivudine in pregnancy for the prevention of perinatal transmission of hepatitis B virus infection. J. Hepatol 55, 1215-1221 (2011). [PubMed: 21703206]

49. Antiretroviral Pregnancy Registry Steering Committee. Antiretroviral Pregnancy Registry International. Interim Report for 1 January through 31 January 2013 Wilmington, NC: Registry Coordinating Center; 2013 [online], http://www.APRegistry.com (2012).

50. Pan CQ et al. Telbivudine prevents vertical transmission from HBeAg-positive women with chronic hepatitis B. Clin. Gastroenterol. Hepatol 10, 520-526 (2012). [PubMed: 22343511]

51. Liu M, Cai H \& Yi W Safety of telbivudine treatment for chronic hepatitis B for the entire pregnancy. J. Viral Hepatol 20 (Suppl. 1), 65-70 (2013).

52. Han GR et al. Long-term safety and efficacy of infants born to telbivudine-treated highly viremic mothers with $\mathrm{HBeAg}$ positive chronic hepatitis B (CHB) during 2nd or 3rd trimester [abstract]. Hepatology 58 (Suppl.), 654A (2013). [PubMed: 23487264]

53. Pan CQ et al. Tenofovir disoproxil fumarate for prevention of vertical transmission of hepatitis $B$ virus infection by highly viremic pregnant women: a case series. Dig. Dis. Sci 57, 2423-2429 (2012). [PubMed: 22543886]

54. Pan C, Liu M, Cai H \& Yo W Safety of tenofovir disoproxil fumarate (TDF) treatment for the entire pregnancy in mothers with active chronic hepatitis B or cirrhosis [abstract]. Hepatology 58 (Suppl.), 624A (2013).

55. Greenup AJ et al. Efficacy and safety of tenofovir in pregnancy to prevent mother to baby transmission of HBV [abstract]. Hepatology 58 (Suppl.), 625A (2013).

56. EASL Clinical Practice Guidelines: Management of chronic hepatitis B virus infection. European Association for the Study of the Liver. J. Hepatol 57, 167-185 (2012). [PubMed: 22436845]

57. Connell LE et al. Maternal hepatitis B and hepatitis C carrier status and perinatal outcomes. Liver Int 31, 1163-1170 (2011). [PubMed: 21745298]

58. Tse KY et al. The impact of maternal HBsAg carrier status on pregnancy outcomes: A casecontrol study. J. Hepatol 43, 771-775 (2005). [PubMed: 16139923]

59. Lao TT et al. Maternal hepatitis B surface antigen and incidence of pre-eclampsia. J. Viral Hepat 20, 343-349 (2013). [PubMed: 23565617]

60. Bzowej NH et al. ALT flares are infrequent during the course of pregnancy: perspectives from the Hepatitis B Research Network (HBRN) adult cohort study [abstract]. Hepatology 58 (Suppl.), 646A-647A (2013).

61. Giles $\mathrm{M}$ et al. Clinical and virological factors that predict post partum flares in pregnant women with chronic, HBV [abstract]. Hepatology 58 (Suppl.), 638A (2013). 
62. Nguyen V et al. Prolonging antiviral therapy after pregnancy for prevention of perinatal HBV transmission does not abrogate post-partum flares [abstract]. Hepatology 58 (Suppl.), 641A (2013).

63. US Department of Health \& Human Services. FDA Viral hepatitis therapies [online], http:// www.fda.gov/ForConsumers/ByAudience/ForPatientAdvocates/ucm151494.htm (2013). 


\section{Review criteria}

A literature search was performed on the PubMed database with the following search terms: "hepatitis B", "pregnancy", "immunoprophylaxis", and "mother-to-child transmission" both alone and in combination. Clinical trials, case series and metaanalyses were analyzed for creation of this Review, with particular focus on manuscripts published in the past 5 years. Abstracts accepted for the 2013 Liver Meeting (published in Hepatology, October 2013) were also reviewed and those pertaining to hepatitis B and pregnancy are included in this Review. 


\section{Key points}

- Perinatal transmission of hepatitis B still occurs worldwide despite the availability of appropriate vaccination

- $\quad$ High maternal HBV viraemia $\left(>10^{7}\right.$ copies per $\left.\mathrm{ml}\right)$ is recognized as a risk factor for immunoprophylaxis failure

- $\quad$ Stratifying the two separate issues of maternal liver disease versus HBV mother-to-child transmission (MTCT) is crucial for clinical decision-making regarding treatment

- $\quad$ Treatment of confirmed high maternal HBV viraemia in the third trimester might be warranted to reduce the risk of MTCT 


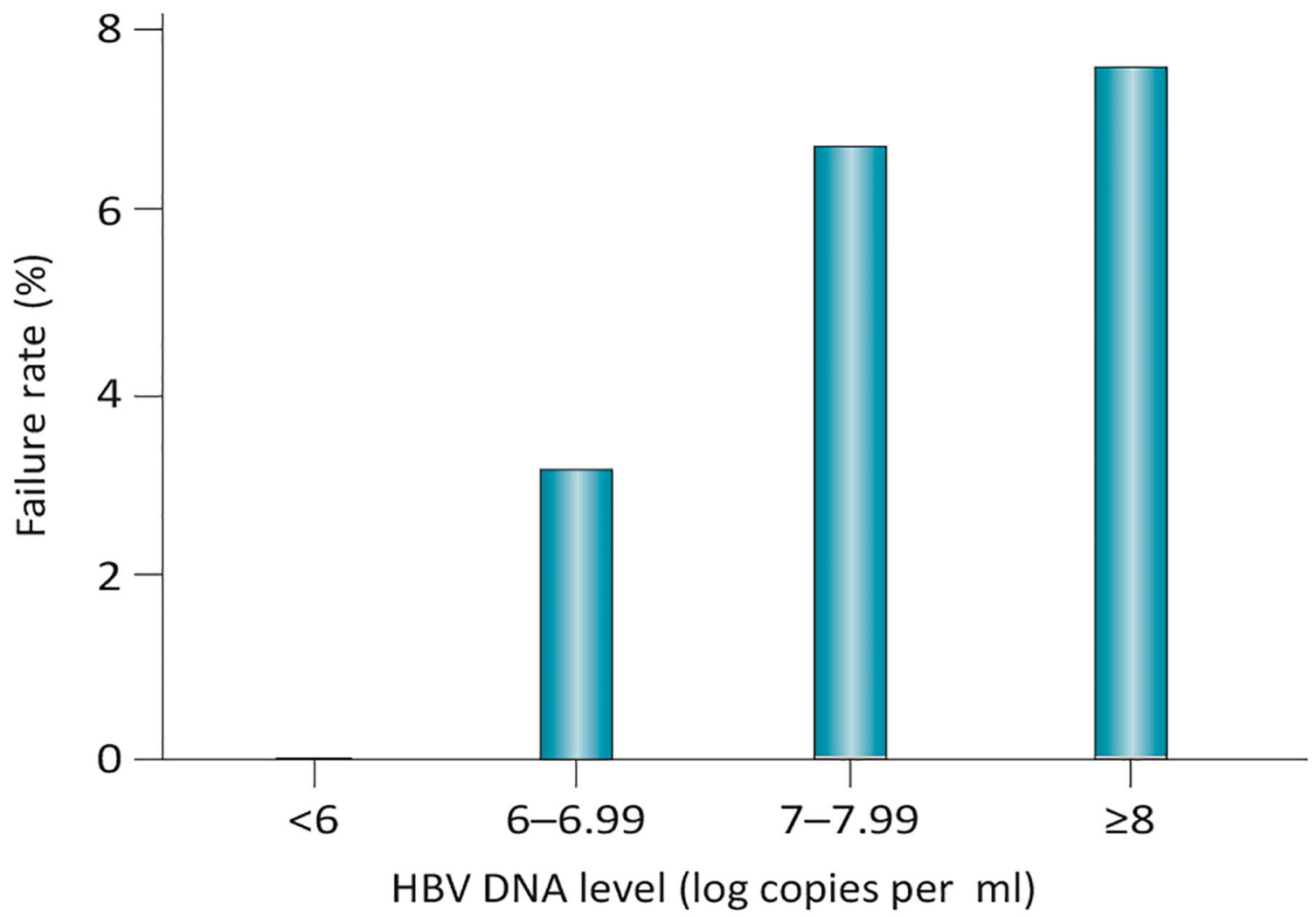

Figure 1 .

Immunoprophylaxis failure rate for HBsAg- positive women according to HBV DNA threshold. All cases of perinatal transmission in this study of 869 women occurred for mothers who were HBeAg-positive and had HBV DNA levels $\ 6 \log _{10}$ copies per $\mathrm{ml}$. Generated using data from Zou et al. ${ }^{35}$ Abbreviations: HBeAg, hepatitis B e antigen; HBsAg, hepatitis B surface antigen. 
Table $1 \mid$

Current FDA-approved therapies for treatment of $\mathrm{HBV}^{63}$

\begin{tabular}{lll}
\hline Generic Name & Indication & $\begin{array}{l}\text { FDA } \\
\text { pregnancy category }\end{array}$ \\
\hline Interferon $a-2 b$ & Chronic HBV, aged $\geq 1$ year, compensated disease & $\mathrm{C}^{*}$ \\
Interferon & $\begin{array}{l}\text { Adult patients with HBV }(\mathrm{HBeAg} \text { positive or negative) with compensated disease, } \\
\text { viral replication and liver inflammation }\end{array}$ \\
Lamivudine & Chronic HBV with viral replication and liver inflammation \\
Adefovir dipivoxil & Chronic HBV, aged $\geq 12$ years \\
Entecavir & Chronic HBV with active viral replication \\
Tenofovir disoproxil fumarate & Chronic HBV in adults & $\mathrm{C}^{*}$ \\
Telbivudine & Chronic HBV in adults with viral replication and transaminitis or histological activity & $\mathrm{B}^{*}$ \\
\hline
\end{tabular}

* Animal reproduction studies have shown an adverse effect on the fetus and there are no adequate and well-controlled studies in humans, but potential benefits may warrant use of the drug in pregnant women despite potential risks.

${ }^{*}$ Animal reproduction studies have failed to demonstrate a risk to the fetus and there are no adequate and well-controlled studies in pregnant women. 


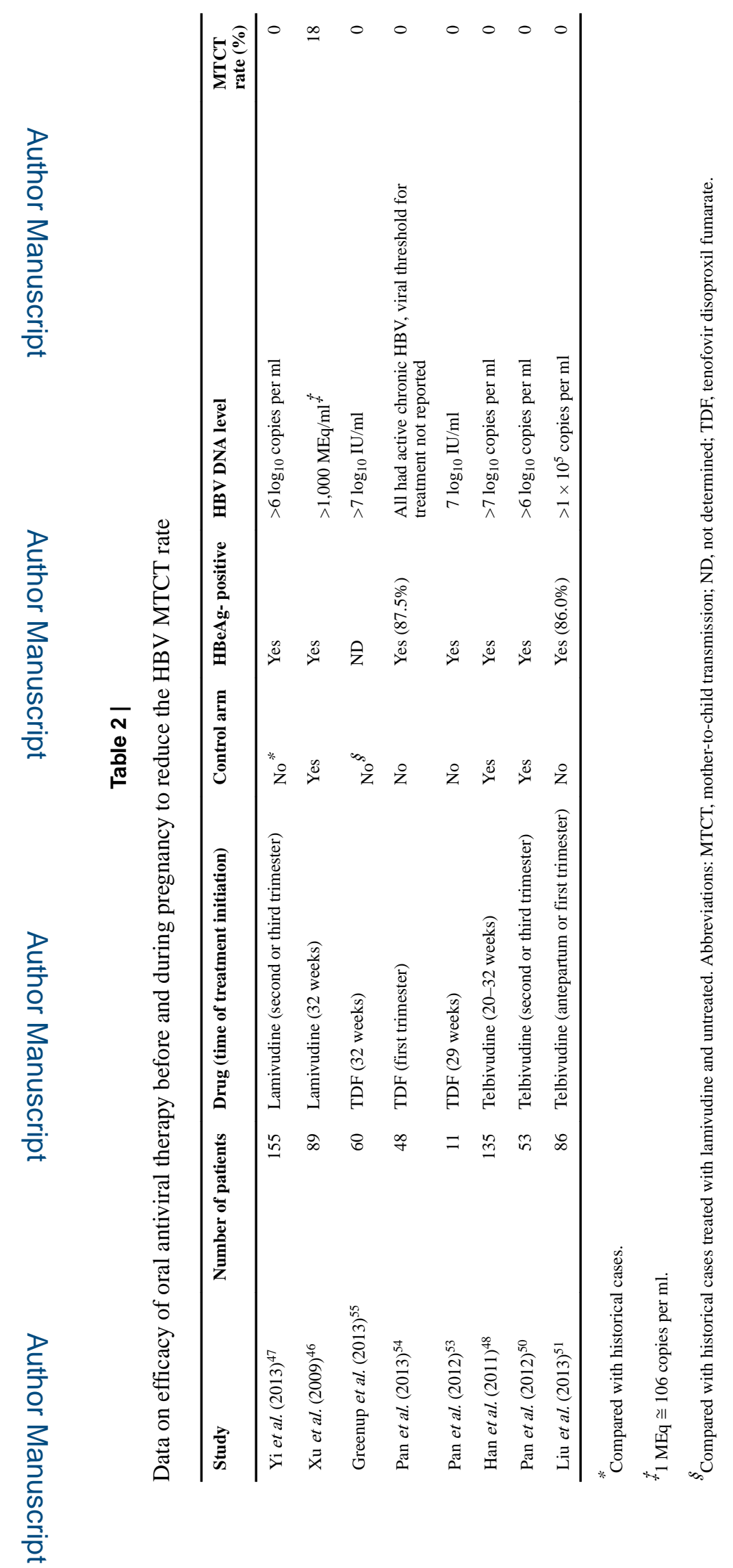

Nat Rev Gastroenterol Hepatol. Author manuscript; available in PMC 2019 July 25. 